\title{
Zoledronic acid in metastatic osteosarcoma: encouraging progression free survival in four consecutive patients
}

\author{
Robert M. Conry ${ }^{1 *}$, Michael G. Rodriguez ${ }^{2}$ and Joseph G. Pressey ${ }^{3,4}$
}

\begin{abstract}
Background: Zoledronic acid (ZA) is a third-generation bisphosphonate in widespread clinical use to reduce pain and skeletal events in patients from a variety of malignancies with bone metastases. Pre-clinical studies indicate that ZA inhibits osteosarcoma through direct anti-proliferative effects, immune activation and anti-angiogenic activity.

Methods: The purpose of this study was to evaluate the antitumor efficacy of ZA at standard dose until progression in patients with stage IV osteosarcoma lacking a standard of care treatment option proven to influence survival. Researchers retrospectively reviewed medical records of all patients at our institution with high-grade osteosarcoma presumed to be incurable due to metastases progressive after primary combination chemotherapy who received single agent ZA in an effort to delay progression.
\end{abstract}

Results: In our four-patient cohort following initiation of ZA, the median progression-free survival was 19 months, and median overall survival was $56+$ months. Two of four patients have remained progression-free since starting ZA. The other two initially progressed after 18-20 months on ZA followed by metastasectomy of lung or dural metastases and further stability for over a year following resumption of ZA. After a 20-month progression-free interval on ZA alone, one patient had partial response following addition of pazopanib to ZA that likely contributed to long term disease control. The four patients experienced no significant toxicities despite protracted dosing of ZA for up to 5 years, and none have required chemotherapy since beginning ZA.

Conclusions: Single agent ZA was associated with encouraging progression-free survival in four consecutive patients with metastatic osteosarcoma. Prospective trials of single agent ZA are warranted as protracted maintenance therapy in surgically incurable osteosarcoma relapsed or refractory to first line combination chemotherapy with radiographically measurable metastases.

Keywords: Zoledronic acid, Bisphosphonates, Osteosarcoma, Metastatic, Survival, Human

\section{Background}

Osteosarcoma is the most common malignant bone tumor in children and young adults. Despite aggressive surgical and medical therapy, the estimated 5-year event-free survivals of patients presenting with localized or metastatic disease are $50-70 \%$ and less than $20 \%$, respectively [1]. Patients with either unresectable primary tumors or distant metastases progressing after

\footnotetext{
*Correspondence: rconry@uabmc.edu

1 Division of Hematology Oncology, University of Alabama

at Birmingham, 2145 Bonner Way, Birmingham, AL 35243, USA

Full list of author information is available at the end of the article
}

conventional primary chemotherapy have dismal outcomes, little changed over the past 30 years [2]. There is no "standard" second-line systemic therapy for inoperable osteosarcoma progressing after conventional chemotherapy. Results of three cooperative group experiences in this population reveal median survival of 6 months [3], median time to progression of 2 months with only $6 \%$ event-free survival at 6 months [4], and a 2-year postrelapse survival $<2 \%$ [5]. Thus, novel systemic treatment approaches are urgently needed.

Zoledronic acid (ZA) is a third-generation, nitrogen-containing bisphosphonate analog of endogenous 
pyrophosphate that strongly binds to calcium-containing hydroxyapatite bone mineral. Although the plasma halflife of ZA is less than $24 \mathrm{~h}$, bisphosphonates are stable in bone with a skeletal half-life of more than 300 days [6]. Osteosarcoma is characterized by the production of osteoid or immature bone containing focal calcium deposits of hydroxyapatite crystals [7]. Thus, ZA may preferentially concentrate in primary osteosarcomas as well as their lung and bone metastases and persist for years. ZA inhibits farnesyl diphosphate synthase, a key enzyme in the mevalonate pathway, and thus reduces protein prenylation essential for normal cell function and survival $[8,9]$. Bone-resorbing osteoclasts internalize relatively large quantities of ZA and are thus particularly vulnerable to functional inhibition, underlying the widespread use of this drug to reduce pain and skeletal-related events in adults with bone metastases [10]. However, nitrogen-containing bisphosphonates appear to have direct anti-proliferative and pro-apoptotic effects against normal osteoblasts and a variety of tumor cells, including breast, myeloma, pancreatic and prostate cancers [11-15]. ZA has been shown to reduce primary tumor growth, decrease lung metastases and prolong survival in animal models of osteosarcoma [16-18]. ZA also acts synergistically with mTOR inhibition to decrease proliferation of murine and human osteosarcoma cell lines in vitro and to reduce osteosarcoma tumor growth in two distinct syngeneic murine modals [19]. Ewing's sarcoma is the second most frequent malignant bone tumor in adolescents and young adults. ZA inhibits Ewing's sarcoma cell invasion through down regulation of matrix metalloproteinase and significantly reduces development of spontaneous lung metastases in a nude mouse model of human Ewing's sarcoma cells injected into the tibia [20].

The Children's Oncology Group (COG) performed a phase I feasibility trial of monthly ZA for eight cycles with concurrent conventional multi-agent chemotherapy in 24 children with previously untreated, high-grade metastatic osteosarcoma [21]. The maximum tolerated dose
(MTD) was equivalent to the standard adult dose for bone metastases, and there was a trend toward improved event-free survival at 2 years compared to historical data from intergroup study INT $0133(p=0.079)$. This manuscript is the first reported clinical experience with ZA as single agent therapy for osteosarcoma.

\section{Methods}

With approval from The University of Alabama at Birmingham institutional review board, the researchers retrospectively reviewed medical records of all patients at our institution with high-grade osteosarcoma treated from December 2010 to January 2015. This timeframe was selected for analysis to allow a minimum of 8 months of follow-up for progression-free survival prior to this report. Twenty-seven such patients were identified; four of whom were presumed to be incurable due to metastatic osteosarcoma progressive after primary combination chemotherapy and received off-label ZA in an effort to delay progression. Those four consecutive patients so treated are the basis of this report. To provide details of each clinical situation and response to $\mathrm{ZA}$, individual patient histories are briefly described in case report format. Treatment outcomes for the four patient cohort are summarized in Table 1.

\section{Results}

All four patients presented with high-grade osteosarcoma without detectable metastases. All received neoadjuvant and/or adjuvant chemotherapy with conventional doses of doxorubicin and cisplatin with or without high-dose methotrexate and ifosfamide. All underwent definitive surgical resection of the primary tumor using a limb-sparing approach in the three extremity cases. Patients 2 and 4 had microscopically positive soft tissue margins treated with post-operative external beam radiotherapy. All patients subsequently developed stage IV osteosarcoma involving the lungs that was treated with ZA. Patients 1 and 2 received ZA as consolidative treatment following metastasectomies and a likely transient

Table 1 Treatment outcomes with zoledronic acid

\begin{tabular}{llllll}
\hline Patient & Sites of disease $^{\mathbf{a}}$ & PFS $^{\mathbf{b}}$ (months) & Additional therapy & Status at last visit & OS $^{\mathbf{b}}$ (months) \\
\hline 1 & Lung & 20 & Pazopanib/surgery & NED & $63+$ \\
2 & Lung & $54+$ & None & NED & $54+$ \\
3 & Lung and hilum & 18 & Radiation to hilum/neurosurgery & SD & $59+$ \\
4 & Lung & $14+$ & None & SD & $14+$ \\
& & & & Median 56+
\end{tabular}

PFS progression-free survival; OS overall survival; NED no evidence of disease; SD stable disease

a sites of osteosarcoma active during or immediately preceding $\mathrm{ZA}$

b calculated from start of ZA 
response to salvage chemotherapy in the case of patient 2. Patients 3 and 4 received ZA for treatment of persistently measurable metastases to the lung and other sites. The University of Alabama at Birmingham Department of Anatomical Pathology histologically confirmed the diagnosis of high-grade osteosarcoma from the primary site of all four patients as well as from resection specimens from lung metastases in patient 1 before and after ZA and patient 2 before ZA. Histological confirmation of metastatic high-grade osteosarcoma was also obtained at our academic center from bronchoscopic biopsy of a hilar lymph node and subsequent resection of a brain metastasis in patient 3 .

\section{Case \#1}

A 20-year-old male presented with osteosarcoma of the left tibia, and 16 months after completion of primary therapy a solitary metastasis of high-grade osteosarcoma was wedge resected from the right upper lobe. Over the subsequent 5 years prior to treatment with $\mathrm{ZA}$, he required bilateral pulmonary metastasectomies on four occasions with no objective response to multiple intervening combination chemotherapy regimens including cyclophosphamide with topotecan, ifosfamide with etoposide, gemcitabine with docetaxel and cisplatin with vinorelbine. During that 5-year period, the longest progression-free interval was 6 months and the interval between metastasectomies progressively shortened from 20 months to 17 to 11 and ultimately to 9 months. A new subpleural $6 \mathrm{~mm}$ left upper lobe metastasis was seen on chest CT performed in June 2010 (Fig. 1a) and markedly enlarged on chest CT in December 2010 (Fig. 1b) prior to his fifth metastasectomy. In an attempt to delay further recurrence, ZA was initiated at $4 \mathrm{mg}$ every 6 weeks rather than a 4-week interval indicated for bone metastases due to lengthy travel to clinic. Chest computed tomographies (CTs) were performed at 3-month intervals. After a 20-month progression-free interval, a solitary $8 \mathrm{~mm}$ metastasis to the left lung was detected (Fig. 1c). The patient continued ZA with the addition of oral pazopanib at $600 \mathrm{mg}$ daily. The solitary metastasis responded to the combination of pazopanib and $\mathrm{ZA}$, decreasing to $3 \mathrm{~mm}$ following 2 months of therapy. After 7 months, pazopanib was reduced to $400 \mathrm{mg}$ daily due to plateaued response (Fig. 1d) as well as mild to moderate anorexia, insomnia and hypertension. After 14 months of combined pazopanib and ZA, chest computed tomography (CT) showed a stable $3 \mathrm{~mm}$ solitary left lung nodule, and ZA was discontinued to facilitate dental procedures related to advanced caries predating ZA use with no evidence of osteonecrosis. Pazopanib was also discontinued 4 months later to facilitate dental work with the lung nodule remaining stable after 18 months of pazopanib

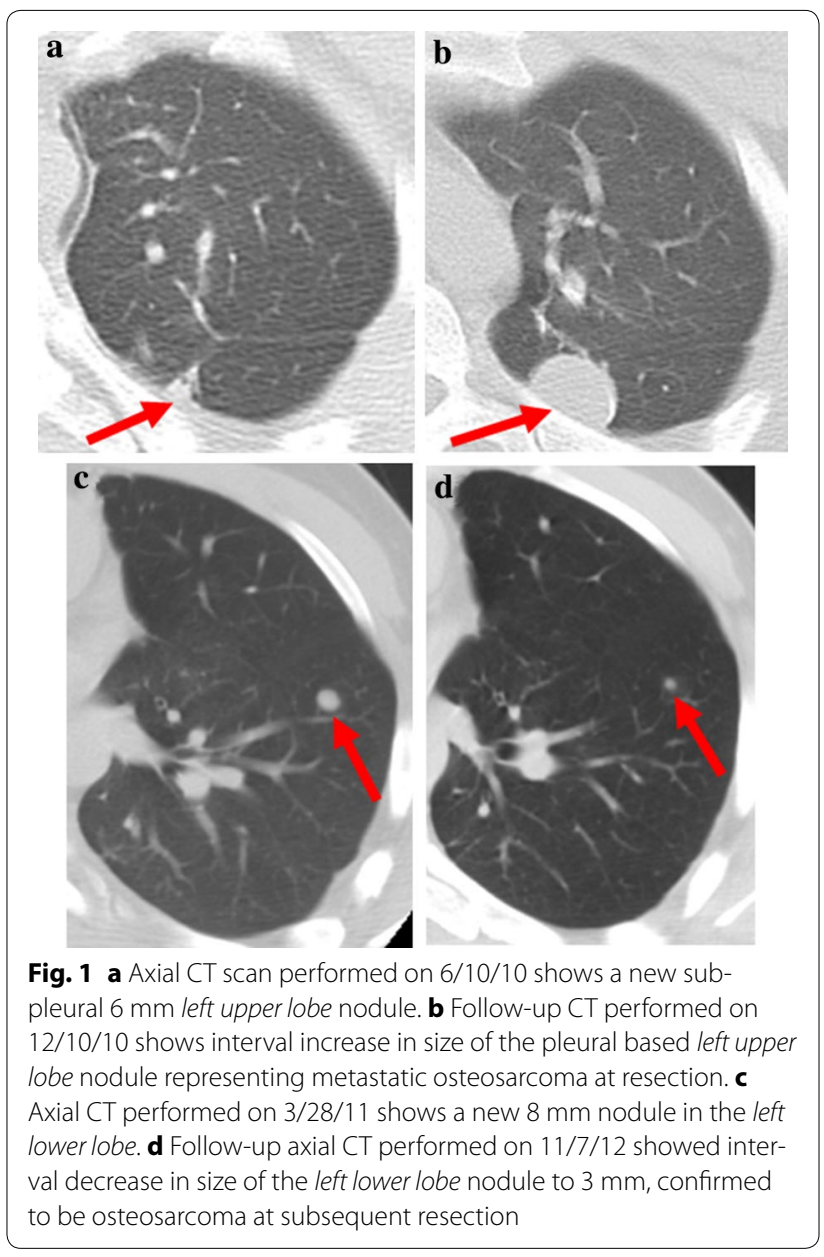

therapy. Chest CT after 3 months off therapy revealed enlargement of the left lung nodule from 3 to $17 \mathrm{~mm}$, and positron emission tomography (PET) showed no disease elsewhere. The solitary left lung metastasis was wedge resected, and single agent ZA was resumed at the prior dose. The patient remains progression-free 20 months after surgery with overall survival $63+$ months from the start of ZA.

\section{Case \#2}

A 63-year-old male underwent left radical parotidectomy and neck dissection to resect an extraosseous osteosarcoma with no lymph node involvement. Two years after completion of primary therapy a CT scan of the chest revealed a new right lung metastasis which was wedge resected. Repeat chest CT scan 3 months later demonstrated five new pulmonary metastases. He was considered incurable due to rapid progression of multiple, bilateral pulmonary metastases 2 years after completion of optimal adjuvant chemotherapy for a man of his age. He received salvage chemotherapy with four cycles of 
doxorubicin and dacarbazine since he was unable to tolerate additional cisplatin due to peripheral neuropathy. Chest CT scan showed a complete radiographic response which was followed by three cycles of vinorelbine with oral cyclophosphamide. Since the prognosis was dismal, the patient began ZA at 4 mg every 6 weeks due to lengthy travel to clinic as consolidative therapy. He continued this schedule for 18 months followed by dosing every 3 months for 36 additional months without apparent toxicity or relapse of osteosarcoma as determined by chest CT scans at 3-month intervals. He has been on ZA for $54+$ months.

\section{Case \#3}

A 48-year-old female presented with osteosarcoma of the right femur, and 38 months after completion of primary therapy a chest CT scan revealed an enlarging $11 \mathrm{~mm}$ right lung nodule, new calcified left hilar adenopathy and narrowing of the lingular bronchus. Bronchoscopic biopsy confirmed metastatic osteosarcoma. Over the subsequent 19 months, she had no objective response to multiple chemotherapy regimens including ifosfamide with etoposide, cyclophosphamide with vinorelbine, gemcitabine, cisplatin and ridaforolimus. Bilateral pulmonary metastases increased in size and number, and left hilar adenopathy increased from 1.9 to $4.1 \mathrm{~cm}$ with distal atelectasis, cough and dyspnea on exertion (Fig. 2a, b). Since standard treatment options had been exhausted, ZA was initiated at $3.5 \mathrm{mg}$ every 4 weeks due to an abnormal glomerular filtration rate at baseline. After 18 months of ZA, bilateral lung metastases remained stable, and the left hilar adenopathy increased modestly to $5 \mathrm{~cm}$ with progressive atelectasis. Although she was stable by RECIST 1.1 criteria, palliative radiation to 40 Gy in 8 fractions was delivered to the hilar mass to reduce dyspnea, and ZA was continued. After 26 additional months of ZA, the hilar adenopathy and multiple, bilateral pulmonary metastases remained stable by RECIST 1.1 criteria. A $5 \mathrm{~cm}$ intracranial dural-based calcified falcine mass was incidentally detected on sinus films, and neurosurgical resection revealed metastatic osteosarcoma. She has remained on monthly ZA for $59+$ months and is stable 16 months after resection of the intracranial metastasis (Fig. 2c, d).

\section{Case \#4}

A 65-year-old male presented with chondroblastic osteosarcoma of the left femur and multiple new bilateral lung metastases measuring up to $1 \mathrm{~cm}$ diameter developed during the fourth cycle of adjuvant chemotherapy. These were confirmed to be progressive by two CT scans of the chest performed 6 weeks apart (Fig. 3a, b).
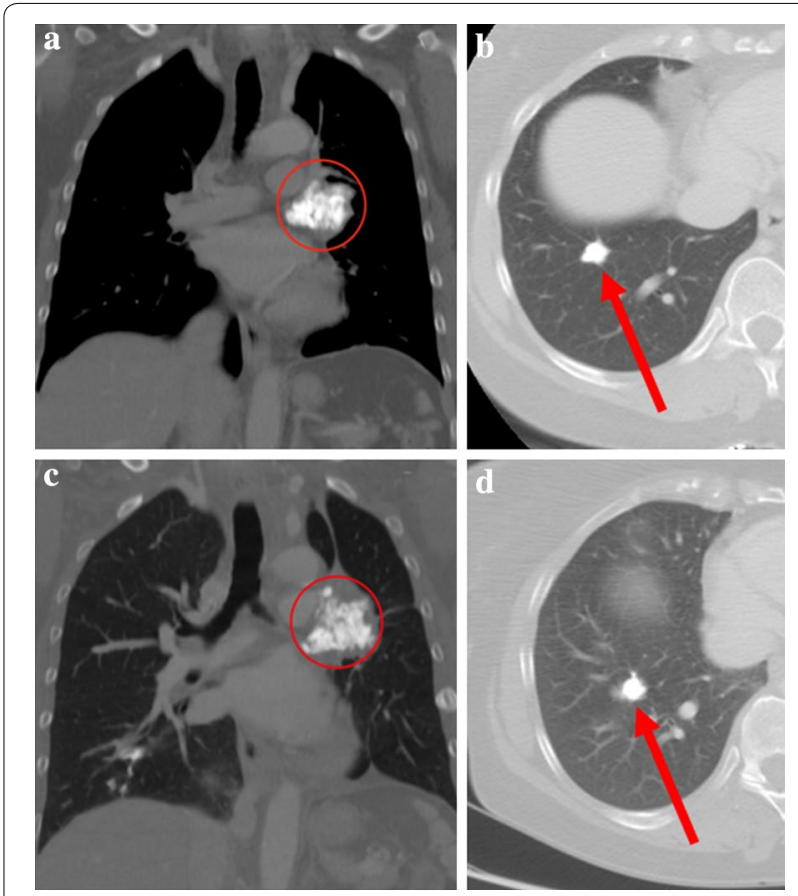

Fig. 2 a Coronal CT image on 3/31/11 showing a calcified left hilar mass with metastatic osteosarcoma biopsy confirmed. b Axial CT image performed on 3/31/11 showing a calcified nodule in the right lower lobe consistent with metastatic disease. c Coronal CT image performed on 12/11/15 shows grossly stable appearance of the calcified left hilar mass. d Axial CT image performed on 12/11/15 shows a grossly stable appearance of the right lower lobe nodule

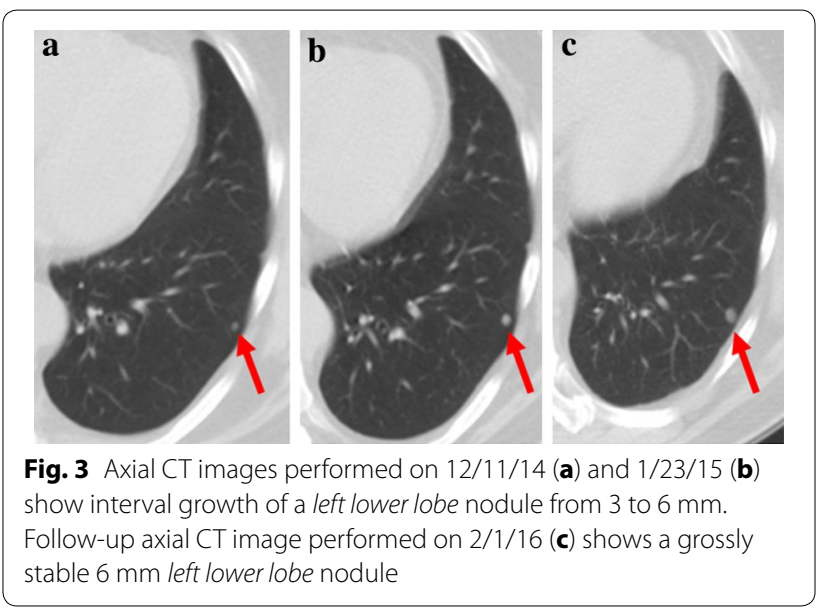

Chemotherapy was discontinued, and ZA was initiated at $4 \mathrm{mg}$ every 4 weeks. After 14 months of follow-up, the patient remains stable with no local recurrence, pulmonary metastases stable by RECIST 1.1 criteria (Fig. 3c) and no new lesions as determined by MRI and chest CT, respectively at 3 month intervals. 


\section{Summary of outcomes}

All patients had stage IV osteosarcoma and lacked a standard of care treatment option proven to significantly influence survival. The treatment outcomes with $\mathrm{ZA}$ are summarized in Table 1. Following initiation of ZA, the median progression free survival was 19 months, and median overall survival was $56+$ months. At the time of this report, two of the four patients have no evidence of disease, and the other two have stable disease. None of the four patients have required chemotherapy since starting ZA. The case of patient 1 is particularly compelling since ZA dramatically extended the progression-free interval following his fifth pulmonary metastasectomy to 20 months. Once another metastasis became detectable, it objectively responded to the addition of pazopanib to ZA for 14 months followed by resection, and he remains progression-free 20 months later on single agent ZA. Patient 3 demonstrated stable hilar and pulmonary metastases for a remarkable 44 months on ZA allowing her to survive long enough to develop a very uncommon metastasis to the dura possibly representing a sanctuary site of relatively poor drug penetration. Following resection of the intracranial metastasis, she has remained stable for an additional 16 months on ZA.

\section{Discussion}

Bisphosphonates are relatively well tolerated with side effects including electrolyte disturbances, flu-like symptoms, nausea and less commonly osteonecrosis of the jaw and renal dysfunction [22, 23]. The four patients reported herein receiving ZA for advanced osteosarcoma experienced no significant toxicities despite protracted dosing for up to 5 years. ZA is a convenient treatment for patients with incurable osteosarcoma with far less detrimental impact on quality of life than conventional chemotherapy.

Pre-clinical studies indicate that ZA inhibits osteosarcoma through direct anti-proliferative effects, immune activation and anti-angiogenic activity. ZA directly inhibits human osteosarcoma cell proliferation and induces apoptosis in vitro [24-26]. $\gamma \delta \mathrm{T}$ cells recognize small phosphoantigens associated with metabolites of bacterial isoprenoid biosynthesis or the mevalonate pathway in eukaryotes [27]. ZA sensitizes tumor cells to $\gamma \delta \mathrm{T}$ cell cytotoxicity through accumulation of mevalonate pathway intermediates within bisphosphonate-treated cells [28]. ZA promotes $\gamma \delta$ T cell proliferation from peripheral blood mononuclear cells of osteosarcoma patients and enhances $\gamma \delta \mathrm{T}$ cell cytotoxicity against human osteosarcoma cells $[29,30]$. Vascular endothelial growth factor-A (VEGF-A) and vascular endothelial growth factor receptor-1 (VEGFR-1) are highly expressed by aggressive osteosarcoma cells, but not in clonally-related less aggressive cell lines [31]. Plasma levels of VEGF-A are elevated in osteosarcoma patients, and ZA decreases circulating levels of VEGF-A in these patients [32, 33]. ZA inhibits tumor-associated angiogenesis not only via direct inhibition of endothelial cells, but also by decreasing VEGF-A expression in osteosarcoma. Furthermore, evidence indicates that $\mathrm{ZA}$ induces apoptosis in aggressive osteosarcoma cells at least partly by inhibiting autocrine stimulation via VEGF-A and VEGFR signaling [31]. Thus, nitrogen-containing bisphosphonates have pleiotropic inhibitory effects on osteosarcoma and tumor-associated endothelial cells.

Inherent limitations in this study include the retrospective nature of the review and the small cohort of patients analyzed. However, this report does include all patients receiving single agent ZA to delay osteosarcoma progression at our institution during the specified timeframe.

COG has demonstrated the feasibility of co-administering ZA with conventional chemotherapy for first line treatment of metastatic osteosarcoma, but the small trial was underpowered to detect efficacy of ZA, particularly in the presence of highly active chemotherapy. A large, randomized French trial of conventional adjuvant chemotherapy with or without ZA for resectable primary osteosarcoma was stopped early due to futility with hazard ratios for event-free survival and death exceeding unity in the ZA cohort. Although not statistically significant, these data suggests that ZA in this context may increase mortality [34]. However, this trial may likewise have been suboptimally designed to evaluate efficacy of ZA in this disease. Direct antiproliferative effects of ZA could actually protect osteosarcoma cells from tumoricidal effects of chemotherapy. Enhancement of antitumor immune responses by ZA could be undermined by concurrent cytotoxic chemotherapy, and antiangiogenic actions of ZA may have little relevance in treating micrometastases in the adjuvant setting. ZA may actually have greater efficacy against surgically incurable macroscopic osteosarcoma used as a single agent or in combination with the mTOR inhibitor everolimus or the VEGFR tyrosine kinase inhibitor pazopanib, targeting tumor cell proliferation and angiogenesis, respectively $[19,35]$. The combination of ZA and sirolimus has recently been demonstrated to be effective and tolerable therapy for a young adult with aggressive lymphatic malformations associated with Gorham-Stout disease [36]. Intriguing results from a recent study of the multi-tyrosine kinase inhibitor sorafenib with activity against VEGFR in combination with everolimus further support the use of such agents in recurrent or refractory osteosarcoma [37]. In fact, this trial represents the first positive study in at least 25 years in patients with relapsed osteosarcoma [38]. Interestingly, patient 1 in this report experienced disease control 
for 20 months on single agent ZA followed by emergence of another lung metastasis that objectively responded for over 14 months to the addition of pazopanib to ZA. We have also observed objective response of multiple pulmonary metastases to combined ZA and pazopanib for over 9 months in a patient with relapsed osteosarcoma not included here due to lack of single agent ZA use.

\section{Conclusions}

Zoledronic acid represents a convenient, well tolerated therapy which may provide durable control of incurable osteosarcoma. Therefore, prospective trials of single agent ZA are warranted in surgically incurable osteosarcoma relapsed or refractory to first line conventional combination chemotherapy with radiographically measurable metastases.

\begin{abstract}
Abbreviations
ZA: zoledronic acid; mTOR: mammalian target of rapamycin; CT: computed tomography; PET: positron emission tomography; RECIST: response evaluation criteria in solid tumors; MRI: magnetic resonance imaging; VEGF: vascular endothelial growth factor; VEGFR: vascular endothelial growth factor receptor; COG: Children's Oncology Group.
\end{abstract}

\section{Authors' contributions}

Dr. RMC was involved in conception of the study, treating patients and monitoring their antitumor response and toxicities. He was actively involved in writing the manuscript. Dr. MGR comprehensively reviewed patient imaging and provided Figs. 1, 2, and 3. Dr. JGP assisted with study conception, data interpretation and manuscript writing. All authors read and approved the final manuscript.

\section{Author details \\ 1 Division of Hematology Oncology, University of Alabama at Birmingham, 2145 Bonner Way, Birmingham, AL 35243, USA. ${ }^{2}$ Department of Radiology, University of Alabama at Birmingham, 619 19th St South, Birmingham, AL 35249, USA. ${ }^{3}$ Department of Pediatrics, University of Alabama at Birmingham, 1600 7th Avenue South, Birmingham, AL 35233, USA. ${ }^{4}$ Present Address: Can- cer \& Blood Disorders Institute, Cincinnati Children's Hospital Medical Center, Cincinnati, OH, USA.}

\section{Acknowledgements}

The authors thank Kendra Gilpin for expert preparation of the manuscript.

\section{Competing interests}

The authors declare that they have no competing interests.

\section{Consent for publication}

Consent has been obtained from the four patients involved and is on file at the University of Alabama at Birmingham.

Received: 23 January 2016 Accepted: 29 March 2016

Published online: 28 April 2016

\section{References}

1. Bacci G, Ferrari S, Bertoni F, Ruggieri P, Picci P, Longhi A, et al. Long-term outcome for patients with nonmetastatic osteosarcoma of the extremity treated at the istituto ortopedico rizzoli according to the istituto ortopedico rizzoli/osteosarcoma-2 protocol: an updated report. J Clin Oncol Off J Am Soc Clin Oncol. 2000;18:4016-27.
2. Grignani G, Palmerini E, Dileo P, Asaftei SD, D'Ambrosio L, Pignochino Y, et al. A phase II trial of sorafenib in relapsed and unresectable highgrade osteosarcoma after failure of standard multimodal therapy: an Italian Sarcoma Group study. Ann Oncol Off J Eur Soc Med Oncol ESMO. 2012;23:508-16

3. Kempf-Bielack B, Bielack SS, Jurgens H, Branscheid D, Berdel WE, Exner GU, et al. Osteosarcoma relapse after combined modality therapy: an analysis of unselected patients in the Cooperative Osteosarcoma Study Group (COSS). J Clin Oncol Off J Am Soc Clin Oncol. 2005;23:559-68.

4. Leary SE, Wozniak AW, Billups CA, Wu J, McPherson V, Neel MD, et al. Survival of pediatric patients after relapsed osteosarcoma: the St. Jude Children's Research Hospital experience. Cancer. 2013;119:2645-53.

5. Ferrari S, Briccoli A, Mercuri M, Bertoni F, Picci P, Tienghi A, et al. Postrelapse survival in osteosarcoma of the extremities: prognostic factors for long-term survival. J Clin Oncol Off J Am Soc Clin Oncol. 2003;21:710-5.

6. Fleisch H. Bisphosphonates. Pharmacology and use in the treatment of tumour-induced hypercalcaemic and metastatic bone disease. Drugs. 1991;42:919-44

7. Mierau GW, Weeks DA, Hicks MJ. Role of electron microscopy and other special techniques in the diagnosis of childhood round cell tumors. Hum Pathol. 1998;29:1347-55.

8. Perry CM, Figgitt DP. Zoledronic acid: a review of its use in patients with advanced cancer. Drugs. 2004;64:1197-211.

9. Green JR. Skeletal complications of prostate cancer: pathophysiology and therapeutic potential of bisphosphonates. Acta Oncol. 2005;44:282-92.

10. Rodan GA, Fleisch HA. Bisphosphonates: mechanisms of action. J Clin Invest. 1996;97:2692-6.

11. Wittrant $Y$, Theoleyre S, Chipoy C, Padrines M, Blanchard F, Heymann D, et al. RANKL/RANK/OPG: new therapeutic targets in bone tumours and associated osteolysis. Biochim Biophys Acta. 2004;1704:49-57.

12. Nakajima H, Magae J, Tsuruga M, Sakaguchi K, Fujiwara I, Mizuta M, et al. Induction of mitochondria-dependent apoptosis through the inhibition of mevalonate pathway in human breast cancer cells by YM529, a new third generation bisphosphonate. Cancer Lett. 2007;253:89-96.

13. Baulch-Brown C, Molloy TJ, Yeh SL, Ma D, Spencer A. Inhibitors of the mevalonate pathway as potential therapeutic agents in multiple myeloma. Leuk Res. 2007;31:341-52.

14. Tassone P, Tagliaferri P, Viscomi C, Palmieri C, Caraglia M, D'Alessandro A, et al. Zoledronic acid induces antiproliferative and apoptotic effects in human pancreatic cancer cells in vitro. Br J Cancer. 2003;88:1971-8.

15. Iguchi K, Nakano T, Usui S, Hirano K. Incadronate inhibits aminopeptidase N expression in prostatic PC-3 cells. Cancer Lett. 2006;237:223-33.

16. Dass CR, Choong PF. Zoledronic acid inhibits osteosarcoma growth in an orthotopic model. Mol Cancer Ther. 2007;6:3263-70.

17. Labrinidis A, Hay S, Liapis V, Ponomarev V, Findlay DM, Evdokiou A. Zoledronic acid inhibits both the osteolytic and osteoblastic components of osteosarcoma lesions in a mouse model. Clin Cancer Res Off J Am Assoc Cancer Res. 2009;15:3451-61.

18. Ory B, Heymann MF, Kamijo A, Gouin F, Heymann D, Redini F. Zoledronic acid suppresses lung metastases and prolongs overall survival of osteosarcoma-bearing mice. Cancer. 2005;104:2522-9.

19. Moriceau G, Ory B, Mitrofan L, Riganti C, Blanchard F, Brion R, et al. Zoledronic acid potentiates mTOR inhibition and abolishes the resistance of osteosarcoma cells to RAD001 (Everolimus): pivotal role of the prenylation process. Cancer Res. 2010;70:10329-39.

20. Odri G, Kim PP, Lamoureux F, Charrier C, Battaglia S, Amiaud J, et al. Zoledronic acid inhibits pulmonary metastasis dissemination in a preclinical model of Ewing's sarcoma via inhibition of cell migration. BMC Cancer. 2014;14:169.

21. Goldsby RE, Fan TM, Villaluna D, Wagner LM, Isakoff MS, Meyer J, et al. Feasibility and dose discovery analysis of zoledronic acid with concurrent chemotherapy in the treatment of newly diagnosed metastatic osteosarcoma: a report from the Children's Oncology Group. Eur I Cancer. 2013:49:2384-91.

22. Bamias A, Kastritis E, Bamia C, Moulopoulos LA, Melakopoulos I, Bozas G, et al. Osteonecrosis of the jaw in cancer after treatment with bisphosphonates: incidence and risk factors. J Clin Oncol Off J Am Soc Clin Oncol. 2005;23:8580-7.

23. Perazella MA, Markowitz GS. Bisphosphonate nephrotoxicity. Kidney Int. 2008;74:1385-93. 
24. Chang J, Wang W, Zhang H, Hu Y, Yin Z. Bisphosphonates regulate cell proliferation, apoptosis and pro-osteoclastic expression in MG-63 human osteosarcoma cells. Oncol Lett. 2012;4:299-304.

25. Ory B, Blanchard F, Battaglia S, Gouin F, Redini F, Heymann D. Zoledronic acid activates the DNA S-phase checkpoint and induces osteosarcoma cell death characterized by apoptosis-inducing factor and endonuclease$\mathrm{G}$ translocation independently of p53 and retinoblastoma status. Mol Pharmacol. 2007;71:333-43.

26. Hirbe AC, Roelofs AJ, Floyd DH, Deng H, Becker SN, Lanigan LG, et al. The bisphosphonate zoledronic acid decreases tumor growth in bone in mice with defective osteoclasts. Bone. 2009;44:908-16.

27. Todaro M, D'Asaro M, Caccamo N, lovino F, Francipane MG, Meraviglia S, et al. Efficient killing of human colon cancer stem cells by gammadelta T lymphocytes. J Immunol. 2009;182:7287-96.

28. D’Asaro M, La Mendola C, Di Liberto D, Orlando V, Todaro M, Spina M, et al. $\vee$ gamma $9 \mathrm{~V}$ delta $2 \mathrm{~T}$ lymphocytes efficiently recognize and kill zoledronate-sensitized, imatinib-sensitive, and imatinib-resistant chronic myelogenous leukemia cells. J Immunol. 2010;184:3260-8.

29. Li Z, Tang J, Sun L, Ye Z. Effect of zoledronate on the cytotoxicity of gammadelta T cells from PBMCs of osteosarcoma patients against osteosarcoma. Chin J Cell Mol Immunol. 2013;29:6-9.

30. Li Z. Potential of human gammadelta T cells for immunotherapy of osteosarcoma. Mol Biol Rep. 2013;40:427-37.

31. Ohba T, Cates JM, Cole HA, Slosky DA, Haro H, Ichikawa J, et al. Pleiotropic effects of bisphosphonates on osteosarcoma. Bone. 2014;63:110-20.
32. Santini D, Vincenzi B, Galluzzo S, Battistoni F, Rocci L, Venditti O, et al. Repeated intermittent low-dose therapy with zoledronic acid induces an early, sustained, and long-lasting decrease of peripheral vascular endothelial growth factor levels in cancer patients. Clin Cancer Res Off J Am Assoc Cancer Res. 2007;13:4482-6.

33. Savitskaya YA, Rico-Martinez G, Linares-Gonzalez LM, Delgado-Cedillo EA, Tellez-Gastelum R, Alfaro-Rodriguez AB, et al. Serum tumor markers in pediatric osteosarcoma: a summary review. Clin Sarcoma Res. 2012;2:9.

34. Battaglia S, Dumoucel S, Chesneau J, Heymann MF, Picarda G, Gouin F, et al. Impact of oncopediatric dosing regimen of zoledronic acid on bone growth: preclinical studies and case report of an osteosarcoma pediatric patient. J Bone Miner Res Off J Am Soc Bone Miner Res. 2011;26:2439-51.

35. Safwat A, Boysen A, Lucke A, Rossen P. Pazopanib in metastatic osteosarcoma: significant clinical response in three consecutive patients. Acta Oncol. 2014;53:1451-4.

36. Cramer SL, Wei S, Merrow AC, Pressey JG. Gorham-Stout disease successfully treated with sirolimus and zoledronic acid therapy. J Pediatr Hematol Oncol. 2016;38:e129-32

37. Grignani G, Palmerini E, Ferraresi V, D’Ambrosio L, Bertulli R, Asaftei SD, et al. Sorafenib and everolimus for patients with unresectable high-grade osteosarcoma progressing after standard treatment: a non-randomised phase 2 clinical trial. Lancet Oncol. 2015;16:98-107.

38. Wagner MJ, Livingston JA, Patel SR, Benjamin RS. Chemotherapy for bone sarcoma in adults. J Oncol Pract Am Soc Clin Oncol. 2016:12:208-16.

\section{Submit your next manuscript to BioMed Central and we will help you at every step:}

- We accept pre-submission inquiries

- Our selector tool helps you to find the most relevant journal

- We provide round the clock customer support

- Convenient online submission

- Thorough peer review

- Inclusion in PubMed and all major indexing services

- Maximum visibility for your research

Submit your manuscript at www.biomedcentral.com/submit

\section{() BioMed Central}

